\title{
Moving Big Data to The Cloud: An Online Cost-Minimizing Approach
}

\author{
Linquan Zhang, Chuan Wu, Zongpeng Li, Chuanxiong Guo, Minghua Chen and Francis C.M. Lau
}

\begin{abstract}
Cloud computing, rapidly emerging as a new computation paradigm, provides agile and scalable resource access in a utility-like fashion, especially for the processing of big data. An important open issue here is to efficiently move the data, from different geographical locations over time, into a cloud for effective processing. The de facto approach of hard drive shipping is not flexible or secure. This work studies timely, cost-minimizing upload of massive, dynamically-generated, geo-dispersed data into the cloud, for processing using a MapReduce-like framework. Targeting at a cloud encompassing disparate data centers, we model a cost-minimizing data migration problem, and propose two online algorithms: an online lazy migration (OLM) algorithm and a randomized fixed horizon control (RFHC) algorithm , for optimizing at any given time the choice of the data center for data aggregation and processing, as well as the routes for transmitting data there. Careful comparisons among these online and offline algorithms in realistic settings are conducted through extensive experiments, which demonstrate close-to-offline-optimum performance of the online algorithms.
\end{abstract}

Index Terms-Cloud Computing, Big Data, Online Algorithms

\section{INTRODUCTION}

The cloud computing paradigm enables rapid on-demand provisioning of server resources (CPU, storage, bandwidth) to users, with minimal management efforts. Recent cloud platforms, as exemplified by Amazon EC2 and S3, Microsoft Azure, Google App Engine, Rackspace [1], etc., organize a shared pool of servers from multiple data centers, and serve their users using virtualization technologies.

The elastic and on-demand nature of resource provisioning makes a cloud platform attractive for the execution of various applications, especially computation-intensive ones [2], [3]. More and more data-intensive Internet applications, e.g., Facebook, Twitter, and big data analytics applications, such as the Human Genome Project [4], are relying on the clouds for processing and analyzing their petabyte-scale data sets, with a computing framework such as MapReduce and Hadoop [5], [6]. For example, Facebook-like social media sites collect

Manuscript received on December 1, 2012; revised on May 28, 2012. Some preliminary results were presented at IEEE INFOCOM 2013, mini-conference, Turin, Italy, April, 2013.

L. Zhang and $\mathrm{Z}$. Li are with the Department of Computer Science, University of Calgary. Email: \{linqzhan, zongpeng\} @ucalgary.ca. C. Wu and F. C.M. Lau are with the Department of Computer Science, The University of Hong Kong. Email: \{cwu, fcmlau\}@cs.hku.hk. C. Guo is with Microsoft. Email: chguo@microsoft.com. M. Chen is with the Department of Information Engineering, The Chinese University of Hong Kong. Email minghua@ie.cuhk.edu.hk.

Work supported by NSERC, Canada (RT733206), RGC, Hong Kong (717812, AoE/E-02/08, 411010, 411011), and National Basic Research Program of China (2012CB315904, 2013CB336700) their Web server logs, Internet click data, social activity reports, etc., from various locations over time, and parse them using Mapreduce/Hadoop to uncover usage patterns and hidden correlations, in order to facilitate marketing decisions.

While most efforts have been devoted to designing better computing models for big data analytics, an important issue has largely been left out in this respect: How does one move the massive amounts of data into a cloud, in the very first place? The current practice is to copy the data into large hard drives for physically transportation to the data center [7], [8], or even to move entire machines [9]. Such physical transportation incurs undesirable delay and possible service downtime, while outputs of the data analysis are often needed to be presented to users in the most timely fashion [9]. It is also less secure, given that the hard drives are prone to infection of malicious programs and damages from road accidents. A safer and more flexible data migration strategy is in need, to minimize any potential service downtime.

The challenge escalates when we target at dynamically and continuously produced data from different geographical locations, e.g., astronomical data from disparate observatories [10], usage data from different Facebook Web servers. With dynamic data, an efficient online algorithm is desired, for timely guiding the transfer of data into the cloud over time. For geo-dispersed data sets, we wish to select the best data center to aggregate all data onto, for processing with a MapReduce-like framework, which is efficient to process data within one data center but not across data centers, due to the enormous overhead of inter-data center data moving in the stage of shuffle and reduce [11]. For example, Amazon Elastic MapReduce launches all processing nodes of a MapReduce job in the same EC2 Availability Zone [12].

As the first dedicated effort in the cloud computing literature, this work studies timely, cost-minimizing migration of massive amounts of dynamically-generated, geo-dispersed data into the cloud, for processing using a MapReduce-like framework. Targeting a typical cloud platform that encompasses disparate data centers of different resource charges, we carefully model the cost-minimizing data migration problem, and propose efficient offline and online algorithms, which optimize the routes of data into the cloud and the choice of the data center for data processing, at any give time. Our detailed contributions are as follows:

$\triangleright$ We analyze the detailed cost composition and identify the performance bottleneck for moving data into the cloud, and formulate an offline optimal data migration problem. The optimization computes optimal data routing and aggregation 
strategies at any given time, and minimizes the overall system cost and data transfer delay, over a long run of the system.

$\triangleright$ We propose a polynomial-time dynamic programming based algorithm to solve the offline optimal data migration problem, given complete knowledge of data generation in the temporal domain. The derived offline optimal strategies serve as a benchmark for our online algorithms.

$\triangleright$ Two efficient online algorithms are proposed to practically guide data migration over time: an online lazy migration (OLM) algorithm and a randomized fixed horizon control (RFHC) algorithm. Theoretical analyses show that the OLM algorithm achieves a worst-case competitive ratio of 2.55 , without the need of any future information and regardless of the system scale, under the typical settings in real-world scenarios. The RFHC algorithm achieves a competitive ratio of $1+\frac{1}{l+1} \frac{\kappa}{\lambda}$ that approaches 1 as the lookahead window $l$ grows. Here $\kappa$ and $\lambda$ are system dependent parameters of similar magnitudes.

$\triangleright$ We conduct extensive experiments to evaluate the performance of our online and offline algorithms, using realworld meteorological data generation traces. The online algorithms can achieve close-to-offline-optimum performance in most cases examined, revealing that the theoretical worst-case competitive ratios are pessimistic, and only correspond to rare scenarios in practice.

In the rest of the paper, we discuss related work in Sec. II, describe the system and problem models in Sec. III, and present the offline and online solutions in Sec. IV and Sec. V respectively. Evaluation results are presented in Sec. VI. Sec. VII concludes the paper.

\section{RELATED WORK}

A series of recent work studies application migration to the cloud. Hajjat et al. [13] develop an optimization model for migrating enterprise IT applications onto a hybrid cloud. Cheng et al. [14] and $\mathrm{Wu}$ et al. [15] advocate deploying social media applications into clouds, for leveraging the rich resources and pay-as-you-go pricing. These projects focus on workflow migration and application performance optimization, by carefully deciding the modules to be moved to the cloud and the data caching/replication strategies in the cloud. The very first question of how to move large volumes of application data into the cloud is not explored.

Few existing work discussed such transfer of big data to the cloud. Cho et al. [16] design Pandora, a cost-aware planning system for data transfer to the cloud provider, via both the Internet and courier services. The same authors [17] later propose a solution to minimize the transfer latency under a budget constraint. Different from our study, they focus on static scenarios with a fixed amount of bulk data to transfer, rather than dynamically generated data; in addition, a single cloud site is considered, while our study pays attention to multiple data centers.

A number of online algorithms have been proposed to address different cloud computing and data center issues. For online algorithms without future information, Lin et al. [18] investigate energy-aware dynamic server provisioning, by proposing a Lazy Capacity Provisioning algorithm with a 3-competitive ratio. Mathew et al. [19] tackle the energyaware load balancing problem in the content delivery networks (CDNs) by an online Hibernate algorithm, which strikes a balance between energy consumption and customer servicelevel agreements (SLAs). However, no theoretical analysis on the performance, in terms of competitive ratio, is provided.

For online algorithms assuming lookahead into the future, Lu and Chen [20] study the dynamic provisioning problem in data centers, and design future-aware algorithms based on the classic ski-rental online algorithm, where the competitive ratios can be significantly improved by exploiting the lookahead information up to the break-even interval . Lin et al. [21] investigate load balancing among geographically-distributed data centers, apply a receding horizon control (RHC) algorithm, and show that the competitive ratio can be reduced substantially by leveraging predicted future information, via relaxing the optimization variables. Due to the unique integer requirement on the decision variables, our problem cannot be transformed into the ski-rental framework, nor relaxed to utilize the RHC framework. Our RFHC algorithm handles the integer restriction via randomization instead.

This work fills gaps in the existing literature on the transfer of large amounts of data to the cloud in the following three aspects: 1 , we focus on dynamically generated data rather than static scenarios with a fixed amount of bulk data; 2, our study pays attention to a cloud with multiple geo-distributed data centers, while existing work often focuses on a single cloud site; 3 , existing techniques used to handle online problems in the cloud do not directly apply in our study, due to the unique structure of our data transfer problem.

\section{The Data Migration Problem}

\section{A. System Model}

We consider a cloud consisting of $K$ geo-distributed data centers in a set of regions $\mathcal{K}$, where $K=|\mathcal{K}|$. A cloud user (e.g., a global astronomical telescope application) continuously produces large volumes of data at a set $\mathcal{D}$ of multiple geographic locations (e.g., dispersed telescope sites). The user connects to the data centers from different data generation locations via virtual private networks (VPNs), with $G$ VPN gateways at the user side and $K$ VPN gateways each collocated with a data center. Let $\mathcal{G}$ denote the set of VPN gateways at the user side, with $G=|\mathcal{G}|$. An illustration of the system is in Fig. 1. A private (the user's) network inter-connects the data generation locations and the VPN gateways at the user side. Such a model reflects typical connection approaches between users and public clouds (e.g., AWS Direct Connect [22], Windows Azure Virtual Network [23]), where dedicated, private network connections are established between a user's premise and the cloud, for enhanced security and reliability, and guaranteed inter-connection bandwidth.

Inter-data centre connections within a cloud are usually dedicated high-bandwidth lines [24]. Within the user's private network, the data transmission bandwidth between a data generation location $d \in \mathcal{D}$ and a VPN gateway $g \in \mathcal{G}$ is large as well. The bandwidth $U_{g i}$ on a VPN link $(g, i)$ from 


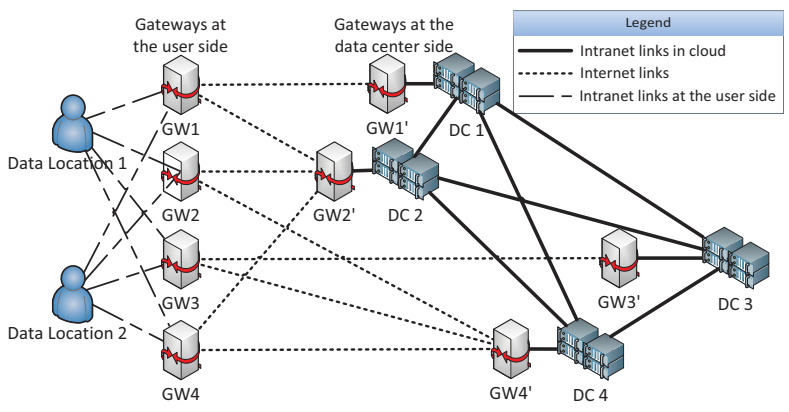

Fig. 1. An illustration of the cloud system.

user side gateway $g$ to data center $i$ is limited, and constitutes the bottleneck in the system.

\section{B. Cost-minimizing Data Migration: Problem Formulation}

Assume the system executes in a time-slotted fashion [15], [18], [19], with slot length $\tau . F_{d}(t)$ bytes of data are produced at location $d$ in slot $t$, for upload to the cloud. $l_{d g}$ is the latency between data location $d \in \mathcal{D}$ and user side gateway $g \in \mathcal{G}$, $p_{g i}$ is the delay along VPN link $(g, i)$, and $\eta_{i k}$ is the latency between data centers $i$ and $k$. These delays, which can be obtained by a simple command such as ping, are dictated by the respective geographic distances.

A cloud user needs to decide (i) via which VPN connections to upload its data to the cloud, and (ii) to which data center to aggregate data, for processing by a MapReduce-like framework, such that the monetary charges incurred, as well as the latency for the data to reach the aggregation point, are jointly minimized. The total cost $\mathbb{C}$ to be minimized has four components: bandwidth cost, aggregate storage and computing cost, migration cost, and routing cost.

Decision variables. Two types of optimization variables are formulated:

(1) Data routing variable $x_{d, g, i, k}(t), \forall d \in \mathcal{D}, \forall g \in \mathcal{G}, \forall i \in$ $\mathcal{K}, \forall k \in \mathcal{K}$, denotes the portion of data $F_{d}(t)$ produced at location $d$ in $t$, to be uploaded through VPN connection $(g, i)$ and then migrated to data center $k$ for processing. $x_{d, g, i, k}(t)>0$ indicates that the data routing path $d \rightarrow$ $g \rightarrow i \rightarrow k$ is employed, and $x_{d, g, i, k}=0$ otherwise. Let

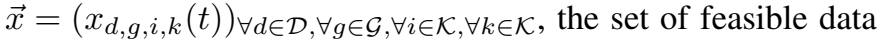
routing variables are:

$$
\begin{array}{r}
\mathcal{X}=\left\{\vec{x}(t) \mid \sum_{g \in \mathcal{G}, i \in \mathcal{K}, k \in \mathcal{K}} x_{d, g, i, k}(t)=1 \text { and } x_{d, g, i, k} \in[0,1],\right. \\
\forall d \in \mathcal{D}, \forall g \in \mathcal{G}, \forall i \in \mathcal{K}, \forall k \in \mathcal{K}\} .
\end{array}
$$

Here $\sum_{g \in \mathcal{G}, i \in \mathcal{K}, k \in \mathcal{K}} x_{d, g, i, k}(t)=1$ ensures that all data produced from location $d$ are uploaded into the cloud in $t$.

(2) Binary variable $y_{k}(t), \forall k \in \mathcal{K}$, indicates whether data center $k$ is target of data aggregation in time slot $t\left(y_{k}(t)=1\right)$ or not $\left(y_{k}(t)=0\right)$. Following the practical requirement of the current MapReduce framework, we require that at any given time, exactly one data center is chosen. Let $\vec{y}(t)=$ $\left(y_{k}(t)\right)_{\forall k \in \mathcal{K}}$, the set of possible data aggregation variables are:

$$
\mathcal{Y}=\left\{\vec{y}(t) \mid \sum_{k \in \mathcal{K}} y_{k}(t)=1 \text { and } y_{k}(t) \in\{0,1\}, \forall k \in \mathcal{K}\right\} .
$$

Costs. The costs incurred in time slot $t$, for uploading the data into the cloud and for processing the data at the selected data center, include the following components.

(1) The overall bandwidth cost for uploading data via the VPN connections, where $\sum_{d \in \mathcal{D}, k \in \mathcal{K}} F_{d}(t) x_{d, g, i, k}(t)$ is the amount uploaded via $(g, i)$, and $f_{g i}$ is the charge for uploading one byte of data via $(g, i)$, derived from bandwidth prices set by the cloud provider:

$$
C_{B W}(\vec{x}(t)) \triangleq \sum_{g \in \mathcal{G}, i \in \mathcal{K}}\left(f_{g i} \sum_{d \in \mathcal{D}, k \in \mathcal{K}} F_{d}(t) x_{d, g, i, k}(t)\right) .
$$

(2) Storage and computation costs are important factors to consider in choosing the data aggregation point. In a large-scale online application, processing and analyzing in $t$ may involve data produced not only in $t$, but also from the past, in the form of raw data or intermediate processing results. For example, data analytics in social networks may re-run the data parsing flow when new data arrives, together using the new data and old data (or previous parsing results of the old data) [25]. Without loss of generality, let the amount of current and history data to process in $t$ be $\mathcal{F}(t)=\sum_{\nu=1}^{t}\left(\alpha_{\nu} \sum_{d \in \mathcal{D}} F_{d}(\nu)\right)$, where $\sum_{d \in \mathcal{D}} F_{d}(\nu)$ is the total amount of data produced in time slot $\nu$ from different data generation locations, and weight $\alpha_{\nu} \in[0,1]$ is smaller for older times $\nu$ and $\alpha_{t}=1$ for the current time $t$. The value of $\alpha_{\nu}$ is determined by specific applications and can be obtained through statistical data. Assume all the other historical data, except those in $\mathcal{F}(t)$, are removed from the data centers where they were processed, since all needed information has been stored in the retained data. Let $\Psi_{k}(\mathcal{F}(t))$ be a non-decreasing cost function for storage and computation in data center $k$ in $t$ (e.g., $\Psi_{k}(\mathcal{F}(t))=s_{k} \mathcal{F}(t)+v_{k} \mathcal{F}(t)$, if $s_{k}$ and $v_{k}$ are the per-byte per-time-slot costs for storing and processing in data center $k$, respectively. The value of $s_{k}$ is determined by the cloud provider and the value of $v_{k}$ depends on both the cloud provider and the specific application). The aggregate storage and computing cost incurred in the cloud in $t$ is:

$$
C_{D C}(\vec{y}(t)) \triangleq \sum_{k \in \mathcal{K}} y_{k}(t) \Psi_{k}(\mathcal{F}(t))
$$

(3) The best data center for data aggregation can differ in $t$ than in $t-1$, due to temporal and spatial variations in data generation. Historical data needed for processing together with new data in $t$, at the amount of $\sum_{\nu=1}^{t-1}\left(\alpha_{\nu} \sum_{d \in \mathcal{D}} F_{d}(\nu)\right)$, should be moved from the earlier data center to the current, and a migration cost is incurred. Let $\phi_{i k}(z)$ be the non-decreasing migration cost to move $z$ bytes of data from data center $i$ to date center $k$, satisfying triangle inequality: $\phi_{i k}(z)+\phi_{k j}(z) \geq$ $\phi_{i j}(z) . \phi_{i k}(z)$ corresponds to upload/download bandwidth charges for moving data out of/into a data center, and/or the delay between the two data centers based on geographic distance. The migration cost between $t-1$ and $t$ is:

$$
\begin{array}{r}
C_{M G}^{t}(\vec{y}(t), \vec{y}(t-1)) \triangleq \sum_{i \in \mathcal{K}} \sum_{k \in \mathcal{K}}\left(\left[y_{i}(t-1)-y_{i}(t)\right]^{+}\right. \\
\left.\left[y_{k}(t)-y_{k}(t-1)\right]^{+} \phi_{i k}\left(\sum_{\nu=1}^{t-1} \alpha_{\nu} \sum_{d \in \mathcal{D}} F_{d}(\nu)\right)\right) .
\end{array}
$$

Here $[a-b]^{+}=\max \{a-b, 0\}$. Since only one data center is selected for data processing in each time slot, there is at most one non-zero term in the above summation, corresponding to 
data centers $i$ for $t-1$ and $k(k \neq i)$ for $t,\left[y_{i}(t-1)-y_{i}(t)\right]^{+}=$ $1=\left[y_{k}(t)-y_{k}(t-1)\right]^{+}=1$.

(4) The latency incurred for data upload is an important performance measure, to be minimized in the data routing and aggregation process. Targeting both monetary cost minimization and delay minimization, we formulate a routing cost for delays along the selected routing paths, and combine it with other costs to be the optimization objective. The overall routing cost in the system in $t$ is:

$$
C_{R T}(\vec{x}(t)) \triangleq \sum_{d, g, i, k} L x_{d, g, i, k}(t) F_{d}(t)\left(l_{d g}+p_{g i}+\eta_{i k}\right),
$$

where $x_{d, g, i, k}(t) F_{d}(t)\left(l_{d g}+p_{g i}+\eta_{i k}\right)$ is the product of data volume and delay along the routing path $d \rightarrow g \rightarrow i \rightarrow k$. The weighted formula suggests that transferring a large volume of data via a high latency path causes high cost. $L$ is the routing cost weight converting $x_{d, g, i, k}(t) F_{d}(t)\left(l_{d g}+p_{g i}+\eta_{i k}\right)$ into a monetary cost, reflecting how latency-sensitive the user is. A cloud user specifies $L$ as a constant a priori. Latency $l_{d g}+p_{g i}+\eta_{i k}$ is fixed in each slot but can change over time.

In summary, the overall cost incurred in $t$ in the system is:

$$
\begin{aligned}
\mathbb{C}(\vec{x}(t), \vec{y}(t))= & C_{B W}(\vec{x}(t))+C_{D C}(\vec{y}(t))+ \\
& C_{M G}^{t}(\vec{y}(t), \vec{y}(t-1))+C_{R T}(\vec{x}(t)) .
\end{aligned}
$$

The offline optimization problem of minimizing the overall cost of data upload and processing over a time interval $[1, T]$ can be formulated as:

$$
\begin{aligned}
& \operatorname{minimize} \sum_{t=1}^{T} \mathbb{C}(\vec{x}(t), \vec{y}(t)) \\
& \text { subject to: } \forall t=1, \ldots, T,
\end{aligned}
$$

(8a) $\vec{x}(t) \in \mathcal{X}$

(8b) $\quad \sum_{d \in \mathcal{K}, k \in \mathcal{K}} F_{d}(t) x_{d, g, i, k}(t) / \tau \leq U_{g i}, \forall i \in \mathcal{K}, \forall g \in \mathcal{G}$,

(8c) $x_{d, g, i, k}(t) \leq y_{k}(t), \forall d \in \mathcal{D}, \forall g \in \mathcal{G}, \forall i \in \mathcal{K}, \forall k \in \mathcal{K}$,

(8d) $\vec{y}(t) \in \mathcal{Y}$,

where $\mathbb{C}(\vec{x}(t), \vec{y}(t)), \mathcal{X}$, and $\mathcal{Y}$ are given in Eqn. (7), (1) and (2), respectively. Constraint $(8 b)$ states that the total amount of data routed via $(g, i)$ into the cloud in each time slot should not exceed the upload capacity of $(g, i)$. (8c) ensures that a routing path $d \rightarrow g \rightarrow i \rightarrow k$ is used $\left(x_{d, g, i, k}(t)>0\right)$, only if data center $k$ is the point of data aggregation in $t\left(y_{k}(t)=1\right)$.

An Online Algorithm takes input gradually as they become available, and computes output on the fly. An Offline Algorithm is given complete information into the future, and generates a solution once in its entirety [26]. Competitive ratio is widely accepted to measure the performance of online algorithms. A deterministic minimizing online algorithm $A$ is $\alpha$-competitive iff $\forall I, A(I) \leq \alpha \cdot O(I)$, where $I$ is a given sequence of input, $A(I)$ is the total cost to complete $I$ by $A, O(I)$ denotes the cost to serve $I$ by the optimal offline algorithm. Similarly, a randomized minimizing online algorithm $A$ is $\alpha$-competitive iff $\forall I, E(A(I)) \leq \alpha \cdot O(I)$.

\section{An Optimal Offline Algorithm}

We first present an offline algorithm, which derives the theoretical minimum cost given complete knowledge of data generation in both temporal and spatial domains. The offline algorithm solves (8) by computing the optimal data routing and aggregation decisions, $\vec{x}^{*}(t)$ and $\vec{y}^{*}(t), t=1, \ldots, T$, using dynamic programming. We construct a 2-D table $W(t, \vec{y}(t))$ that denotes the minimum cost (Eqn. (7)) incurred to upload and process the data $F_{d}(1), F_{d}(2), \ldots, F_{d}(t), \forall d \in \mathcal{D}$, into the cloud, while ending with a specific $\vec{y}(t) \in \mathcal{Y}$ as the data aggregation decision at time $t$. Consider set $\mathcal{Y}$ defined in (2), which in fact has exactly $K$ elements, corresponding to $K$ possible data aggregation decisions at any time $t$. Each data aggregation decision corresponds to selecting exactly one of the $K$ data centers for data processing. Let vector $\vec{y}_{i}$ denote the decision in which data center $i$ is chosen, with $y_{i}(t)=1$ and $y_{j}(t)=0, \forall j \neq i$. Hence $\mathcal{Y}=\left\{\vec{y}_{1}, \vec{y}_{2}, \ldots, \vec{y}_{K}\right\}$. The table $W(t, \vec{y}(t))$ has $K$ rows corresponding to $K$ possible data aggregation decisions in $\mathcal{Y}$, and $T$ columns corresponding to $t=1, \ldots, T$, (Fig. 2).

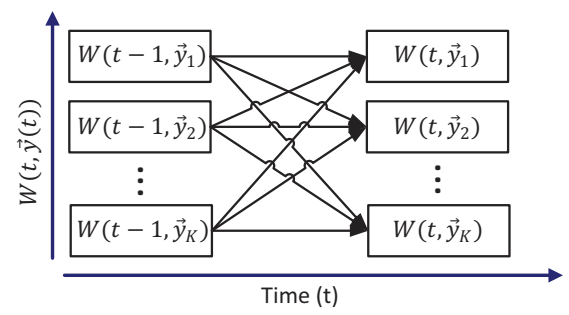

Fig. 2. An illustration of the offline dynamic programming algorithm.

The algorithm begins with $W(0, \vec{y}(0))=0, \forall \vec{y}(0) \in \mathcal{Y}$, and computes the entries inductively:

$$
W(t, \vec{y}(t))=\min _{\substack{\vec{y} \in \mathcal{Y}, \vec{x}(t):(8 \mathrm{a}) \text {-(8c) }}}\{W(t-1, \vec{y})+\mathbb{C}(\vec{x}(t), \vec{y}(t))\} .
$$

Given $\vec{y}(t)$ (data aggregation decision in $t$ ), $W(t, \vec{y}(t))$ computes the minimum cumulative cost from time slot 1 up to $t$, by choosing among all possible data aggregation decisions $\vec{y} \in \mathcal{Y}$ in $t-1$, and all feasible data routing decisions $\vec{x}(t)$ in $t$. The term $W(t-1, \vec{y})$ is the minimum cumulative cost in $[1, t-1]$ with the specific $\vec{y}$ as the data aggregation decision at $t-1$; the term $\mathbb{C}(\vec{x}(t), \vec{y}(t))$, computed by Eqn. (7), is the cost incurred in time slot $t$. Here $\vec{y}$ is related to $\mathbb{C}(\vec{x}(t), \vec{y}(t))$, since it decides the potential migration cost $C_{M G}(\vec{y}(t), \vec{y})$ in $\mathbb{C}(\vec{x}(t), \vec{y}(t))$. If we cannot find a feasible solution to the minimization problem in (9), we set $W(t, \vec{y}(t))=+\infty$.

The rationale of the dynamic programming approach (Alg. 1 ) is as follows. At each time $t$, fixing the choice of the data center used for data processing, we trace back and examine each of the $K$ possible data center choices in time $t-1$, by adding the cost incurred in $t$ to the minimum cumulative cost up to $t-1$; that is, we compute the cumulative costs up to $t$ in $K$ cases, that the historical data are migrated from different data center $j \in \mathcal{K}$ in $t-1$ to $i$ in $t$, supposing data center $i$ is selected in $t$. We then decide the minimum cumulative cost up to time $t$, via the best data center $j$ in $t-1$. Eventually when the table is completed, the minimum overall cost of the system in $[1, T]$, i.e., the optimal objective function value of the offline optimization problem in (8), is given by $\min _{\vec{y}(T) \in \mathcal{Y}} W(T, \vec{y}(T))$. The optimal data aggregation decision in time slot $T$ is $\vec{y}^{*}(T)=\arg \min _{\vec{y}(T) \in \mathcal{Y}} W(T, \vec{y}(T))$, and the optimal data routing decision $\vec{x}^{*}(T)$ is the one leading 


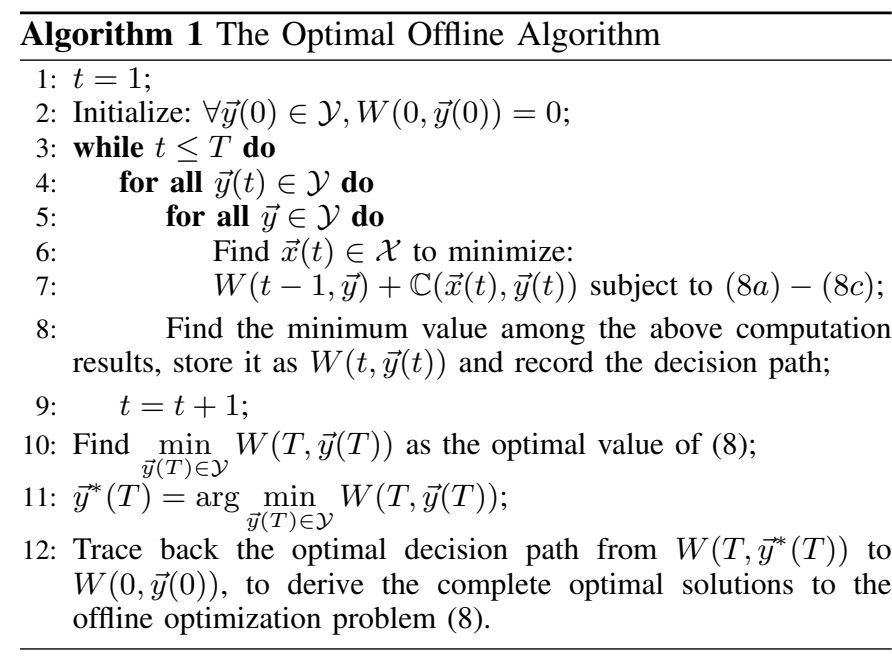

to $W\left(T, \vec{y}^{*}(T)\right)$ by solving (9). The optimal data routing and aggregation decisions in previous time slots can be derived accordingly, by tracing the optimal decision path in the table.

Theorem 1. Alg. 1 has a worst-case computation complexity of $O\left(T \gamma D^{3} G^{3} K^{8} / \ln \left(D G K^{2}\right)\right)$, where $\gamma$ is the number of bits required to represent the following linear program:

$$
\min _{\vec{x}(t)} \mathbb{C}(\vec{x}(t), \vec{y}(t))=C_{B W}(\vec{x}(t))+C_{R T}(\vec{x}(t))
$$

subject to: constraints $(8 a),(8 b),(8 c)$.

Proof sketch: To compute each $W(t, \vec{y}(t))$ using (9), we can loop through the $K$ possible values of $\vec{y} \in \mathcal{Y}$, and when $\vec{y}$ is fixed, the minimization problem reduces to the linear program in (10). For a linear program, efficient polynomialtime algorithms exist, with the current best worst-case complexity of $O\left(\left[n^{3} / \ln n\right] \gamma\right)$ by an interior-point method, where $n$ is the number of variables and $\gamma$ is the number of bits required to represent the linear program [27], related to the numbers of variables and constraints and the number of bits it takes to represent a constant in the LP. There are $D G K^{2}$ variables, $x_{d, g, i, k}(t)$ 's, in this problem, leading to complexity $O\left(\gamma D^{3} G^{3} K^{6} / \ln \left(D G K^{2}\right)\right)$. Therefore, the complexity to compute each $W(t, \vec{y}(t))$ is $O\left(K \cdot \gamma D^{3} G^{3} K^{6} / \ln \left(D G K^{2}\right)\right)=$ $O\left(\gamma D^{3} G^{3} K^{7} / \ln \left(D G K^{2}\right)\right)$. There are $K T$ entries in the table, and hence the overall computation complexity of the optimal offline algorithm is $O\left(T \gamma D^{3} G^{3} K^{8} / \ln \left(D G K^{2}\right)\right)$.

\section{Two Online Algorithms}

\section{A. The Online Lazy Migration (OLM) Algorithm}

The offline optimization problem in (8) can be divided into $T$ one-shot optimization problems:

$$
\text { minimize } \mathbb{C}(\vec{x}(t), \vec{y}(t)) \quad \text { subject to: }(8 a)(8 b)(8 c)(8 d) \text {. }
$$

A straightforward algorithm solves the above optimization in each time slot, based on $\vec{y}(t-1)$ in the previous time slot. This can be far from optimal due to premature data migration. For example, assume data center $k$ was selected at $t-1$, and migrating data from $k$ to $j$ is cost-optimal at $t$ according to the one-shot optimization (e.g., because more data are generated in region $j$ in $t$ ); the offline optimum may indicate to keep all data in $k$ at $t$, if the volume of data generated in $k$ in $t+1$ surges. We next explore dependencies among the selection of the aggregation data center across consecutive time slots, and design a more judicious online algorithm accordingly.

We divide the overall cost $\mathbb{C}(\vec{x}(t), \vec{y}(t))$ incurred in $t$ into two parts: (i) migration cost $C_{M G}^{t}(\vec{y}(t), \vec{y}(t-1))$ defined in (5), related to decisions in $t-1$; (ii) non-migration cost that relies only on current information at $t$ :

$$
C_{-M G}^{t}(\vec{x}(t), \vec{y}(t))=C_{B W}(\vec{x}(t))+C_{D C}(\vec{y}(t))+C_{R T}(\vec{x}(t)) .
$$

We design a lazy migration algorithm (Alg. 2), whose basic idea is to postpone data center switching even if the one-shot optimum indicates so, until the cumulative non-migration cost (in $C_{-M G}^{t}(\vec{x}(t), \vec{y}(t))$ ) has significantly exceeded the potential data migration cost.

At the beginning $(t=1)$, we solve the one-shot optimization in (11), and upload data via the derived optimal routes $\vec{x}(1)$ to the optimal aggregation data center indicted by $\vec{y}(1)$. Let $\hat{t}$ be the time of the data center switch. In each following time slot $t$, we compute the overall non-migration cost in $[\hat{t}, t-1], \sum_{\nu=\hat{t}}^{t-1} C_{-M G}^{\nu}(\vec{x}(\nu), \vec{y}(\nu))$. The algorithm checks whether this cost is at least $\beta_{2}$ times the migration cost $C_{M G}^{\hat{t}}(\vec{y}(\hat{t}), \vec{y}(\hat{t}-1))$. If so, it solves the one-shot optimization to derive $\vec{x}(t)$ and $\vec{y}(t)$ without considering the migration cost, i.e., by minimizing $C_{-M G}^{t}(\vec{x}(t), \vec{y}(t))$ subject to $(8 a)-(8 d)$ and an additional constraint, that the potential migration cost, $C_{M G}^{t}(\vec{y}(t), \vec{y}(t-1))$, is no larger than $\beta_{1}$ times the nonmigration cost $C_{-M G}^{t}(\vec{x}(t), \vec{y}(t))$ at time $t$ (to make sure that the migration cost is not too excessive). If a change of migration data center is indicated $(\vec{y}(t) \neq \vec{y}(t-1))$, the algorithm accepts the new aggregation decision, and migrates data accordingly. In all other cases, the aggregation data center remains unchanged from $t-1$, while optimal data routing paths are computed given this aggregation decision, for upload of new data generated in $t$.

Alg. 2 avoids aggressive switches of the aggregation data center, to prevent moving a large amount of data back and forth too often. Excessive "laziness" is also avoided. Parameters $\beta_{2}>0$ and $\beta_{1}>0$ control the "laziness" and "aggressiveness" of the algorithm: a large $\beta_{2}$ prolongs the inter-switch interval, while a large $\beta_{1}$ invites more frequent switches.

Lemma 1. The overall migration cost in $[1, t]$ is at most $\max \left\{\beta_{1}, 1 / \beta_{2}\right\}$ times the overall non-migration cost in this period, i.e., $\sum_{\nu=1}^{t} C_{M G}^{\nu}(\vec{y}(\nu), \vec{y}(\nu-1)) \leq$ $\max \left\{\beta_{1}, 1 / \beta_{2}\right\} \sum_{\nu=1}^{t} C_{-M G}^{\nu}(\vec{x}(\nu), \vec{y}(\nu))$.

Proof sketch: Potential migration cost in $t$ is at most $\beta_{1}$ times the non-migration costs. For migration cost incurred in previous time slots, i.e., $C_{M G}^{\hat{t}_{i}}\left(\vec{x}\left(\hat{t}_{i}\right), \vec{y}\left(\hat{t}_{i}\right)\right)$ where $\hat{t}_{i}$ is the time of aggregation data center switch, $\forall i=1,2, \cdots$, the nonmigration cost in the period from when this migration occurs to the time before the next migration, i.e., in $\left[\hat{t}_{i}, \hat{t}_{i+1}-1\right]$, is at least $\beta_{2}$ times the migration cost. Hence we have 


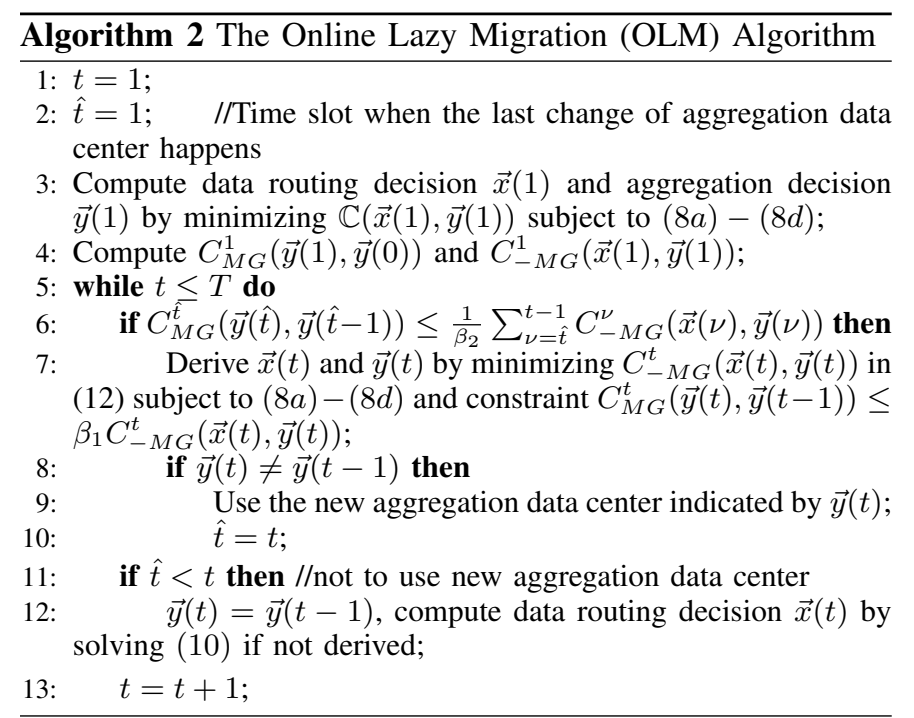

$$
\begin{aligned}
& \sum_{\nu=1}^{t} C_{M G}^{\nu}(\vec{y}(\nu), \vec{y}(\nu-1)) \\
& \leq 1 / \beta_{2} \sum_{\nu=1}^{t-1} C_{-M G}^{\nu}(\vec{x}(\nu), \vec{y}(\nu))+\beta_{1} C_{-M G}^{t}(\vec{x}(t), \vec{y}(t)) \\
& \leq \max \left\{\beta_{1}, 1 / \beta_{2}\right\} \sum_{\nu=1}^{t} C_{-M G}^{\nu}(\vec{x}(\nu), \vec{y}(\nu)) .
\end{aligned}
$$

Lemma 2. The overall non-migration cost in $[1, t]$ is at most $\epsilon$ times the total offline-optimal cost, i.e., $\sum_{\nu=1}^{t} C_{-M G}^{\nu}(\vec{x}(\nu), \vec{y}(\nu)) \leq \epsilon \sum_{\nu=1}^{t} \mathbb{C}\left(\vec{x}^{*}(\nu), \vec{y}^{*}(\nu)\right)$, where

$$
\epsilon=\max _{\nu \in[1, T]} \frac{\max _{\vec{y}(\nu) \in \mathcal{Y}, \vec{x}(\nu):(8 a)-(8 c)} C_{-M G}^{\nu}(\vec{x}(\nu), \vec{y}(\nu))}{\min _{\vec{y}(\nu) \in \mathcal{Y}, \vec{x}(\nu):(8 a)-(8 c)} C_{-M G}^{\nu}(\vec{x}(\nu), \vec{y}(\nu))}
$$

is the maximum ratio of the largest over the smallest possible non-migration cost incurred in a time slot, with different data upload and aggregation decisions.

Proof sketch: By the definition of $\epsilon$, at $\nu \in[1, t]$, the non-migration cost of OLM is smaller than $\epsilon$ times the nonmigration cost incurred by the optimal offline algorithm, i.e., $C_{-M G}^{\nu}(\vec{x}(\nu), \vec{y}(\nu)) \leq \epsilon C_{-M G}^{\nu}\left(\vec{x}^{*}(\nu), \vec{y}^{*}(\nu)\right)$. We have

$$
\begin{aligned}
& \sum_{\nu=1}^{t} C_{-M G}^{\nu}(\vec{x}(\nu), \vec{y}(\nu)) \\
& \leq \epsilon \sum_{\nu=1}^{t} C_{-M G}^{\nu}\left(\vec{x}^{*}(\nu), \vec{y}^{*}(\nu)\right) \\
& \leq \epsilon \sum_{\nu=1}^{t}\left\{C_{-M G}^{\nu}\left(\vec{x}^{*}(\nu), \vec{y}^{*}(\nu)\right)+C_{M G}^{\nu}\left(\vec{y}^{*}(\nu), \vec{y}^{*}(\nu-1)\right)\right\} \\
& =\epsilon \sum_{\nu=1}^{t} \mathbb{C}\left(\vec{x}^{*}(\nu), \vec{y}^{*}(\nu)\right) .
\end{aligned}
$$

Theorem 2. The OLM Algorithm is $\epsilon\left(1+\max \left\{\beta_{1}, 1 / \beta_{2}\right\}\right)$ competitive.

Proof Sketch: The overall cost incurred by the OLM Algorithm in $[1, T]$ is $\sum_{\nu=1}^{T}\left[C_{M G}^{\nu}(\vec{y}(\nu), \vec{y}(\nu-1))+\right.$ $\left.C_{-M G}^{\nu}(\vec{x}(\nu), \vec{y}(\nu))\right]$. By Lemma 1 and Lemma 2:

$$
\begin{aligned}
& \sum_{\nu=1}^{T}\left[C_{M G}^{\nu}(\vec{y}(\nu), \vec{y}(\nu-1))+C_{-M G}^{\nu}(\vec{x}(\nu), \vec{y}(\nu))\right] \\
& \leq\left(1+\max \left\{\beta_{1}, 1 / \beta_{2}\right\}\right) \sum_{\nu=1}^{T} C_{-M G}^{\nu}(\vec{x}(\nu), \vec{y}(\nu)) \\
& \leq \epsilon\left(1+\max \left\{\beta_{1}, 1 / \beta_{2}\right\}\right) \sum_{\nu=1}^{T} \mathbb{C}\left(\vec{x}^{*}(\nu), \vec{y}^{*}(\nu)\right) .
\end{aligned}
$$

The value of $\epsilon$ is mainly determined by data generation patterns over time, and less involved with the system size, e.g., the number of data centers. In our experiments with a typical data generation pattern, $\epsilon=1.7$. In this case, setting $\beta_{1}=0.5$ and $\beta_{2}=2$ leads to a competitive ratio of 2.55 . The competitive ratio is the worst-case performance of the algorithm [26], assuming an adversary who knows the algorithm and chooses the 'worst' input over time. Simulations in Sec. VI reveals much better performance in practice.

Theorem 3. The OLM Algorithm has a computational complexity of $O\left(T \gamma D^{3} G^{3} K^{7} / \ln \left(D G K^{2}\right)\right)$.

Proof Sketch: The worst case of the OLM algorithm occurs when data routing and aggregation decisions need to be derived according to line 7 in Algorithm 2 in each time slot, which is the most time-consuming step in the algorithm. The computation in line 7 can be decomposed into two steps: 1) to minimize $C_{-M G}^{t}(\vec{x}(t), \vec{y}(t))$ in (12) subject to $(8 a)-(8 d)$; 2) to verify constraint $C_{M G}^{t}(\vec{y}(t), \vec{y}(t-1)) \leq \beta_{1} C_{-M G}^{t}(\vec{x}(t), \vec{y}(t))$. The first step requires solving a linear program, with computation complexity of $O\left(\gamma D^{3} G^{3} K^{6} / \ln \left(D G K^{2}\right)\right)$; to complete the second step, it loops through the $K$ possible data aggregation decisions. Therefore the OLM algorithm has a worst-case computation complexity of:

$O\left(T \cdot \gamma D^{3} G^{3} K^{6} / \ln \left(D G K^{2}\right) \cdot K\right)=O\left(T \gamma D^{3} G^{3} K^{7} / \ln \left(D G K^{2}\right)\right)$

The complexity in Theorem 3 is regarding the total running time from $t=1$ to $t=T$. Considering that the OLM algorithm is an online algorithm producing partial results per time slot, we are more interested in the amortized per-slot complexity,which is $O\left(\gamma D^{3} G^{3} K^{7} / \ln \left(D G K^{2}\right)\right)$.

\section{B. The Randomized Fixed Horizon Control (RFHC) Algorithm}

In practical applications, near-term future data generation patterns can often be estimated from history, e.g., using a Markov chain model or a time series forecasting model [28]. We next design an algorithm that exploits such predicted future information. Details in the prediction module is treated as a blackbox, is free to vary, and is not of primary concern in this work. We assume that the information in the lookahead window can be predicted precisely without error.

We divide time into equal-size frames of $l+1$ time slots each $(l \geq 0)$. In the first time slot $t$ of each frame, assume information on data generation for the next $l$ time slots, i.e., $F_{d}(t), F_{d}(t+1), \ldots, F_{d}(t+l), \forall d \in \mathcal{D}$, are known. We solve the following cost minimization over time frame $[t, t+l]$, given the data aggregation decision of $\vec{y}(t-1)$, to derive data routing 


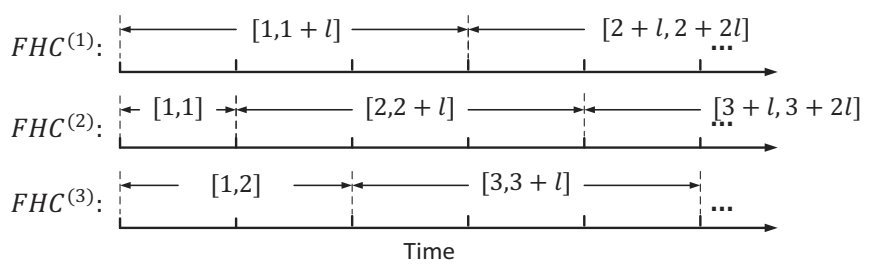

Fig. 3. An illustration of different FHC algorithms with $l=2$.

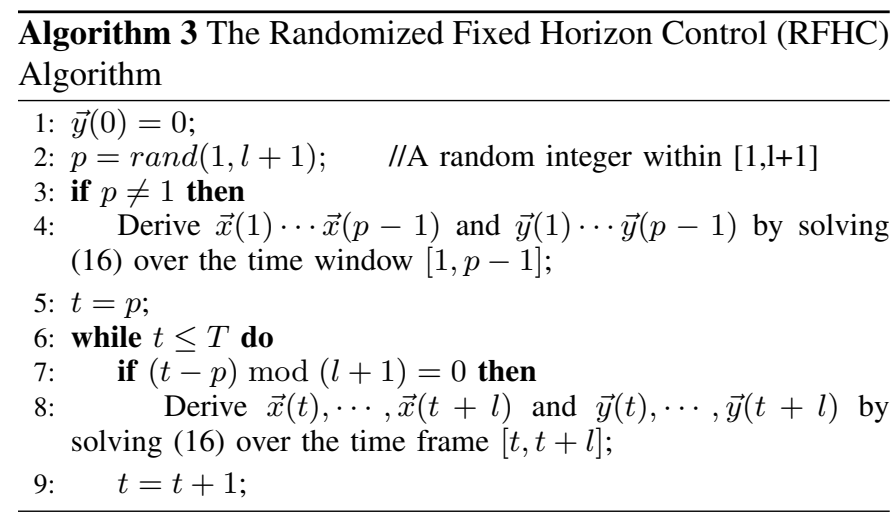

decisions $\vec{x}(\nu)$ and aggregation decisions $\vec{y}(\nu), \forall \nu=t, \ldots, t+$ $l$, using Alg. 1 in Sec. IV:

$$
\text { minimize } \sum_{\nu=t}^{t+l} \mathbb{C}(\vec{x}(\nu), \vec{y}(\nu)),
$$

subject to: constraints (8a)-(8d), for $\nu=t, \ldots, t+l$.

The method is essentially a fixed horizon control (FHC) algorithm, adapted from receding horizon control in the dynamic resource allocation literature [21]. Allowing the first time frame to start from different initial times $p \in[1, l+1]$, we have $l+1$ versions of the FHC algorithm (Fig. 3). In particular, for an algorithm $F H C^{(p)}$ starting from a specific time slot $p$, the above optimization is solved at times $t=p, p+l+1$, $p+2(l+1), \ldots$, for routing and aggregation decisions in the following $l+1$ time slots. For each specific algorithm $F H C^{(p)}$, an adversary can choose an input with a surge of data produced at the beginning of each time frame, leading to a high migration cost. A randomized algorithm defeats such purposeful adversaries, by randomizing the starting times of the frames, achieving lower expected worst-case performance [26].

We design a Randomized Fixed Horizon Control (RFHC) algorithm (Alg. 3). At the beginning, the algorithm uniformly randomly chooses $p \in[1, l+1]$ as the start of the first time frame of $l+1$ slots, i.e., it randomly picks one specific algorithm $F H C^{(p)}$ from the $l+1$ FHC algorithms: at $t=1$, it solves (16) to decide the optimal data routing and aggregation strategies in the period of $t=1$ to $p-1(p \neq 1)$; then at $t=p, p+l+1, p+2(l+1), \ldots$, it solves (16) for optimal strategies in the following $l+1$ time slots, respectively. An adversary, with no information on $p$, finds it hard to contrive specific inputs to degrade the performance of RFHC.

Lemma 3. The overall cost incurred by $F H C^{(p)}$ algorithm is upper-bounded by the offline-optimal cost plus the migration costs to move data from the aggregation data center computed by $F H C^{(p)}$ to that decided by Alg. 1, at the end of the time frames. That is, letting $\vec{x}^{(p)}$ and $\vec{y}^{(p)}$ be the solution derived by the $F H C^{(p)}$ algorithm and $\Omega_{p, t}=\{\omega \mid \omega=p+k(l+1), k=$ $\left.0,1, \ldots,\left\lfloor\frac{t-p}{l+1}\right\rfloor\right\}$, we have for any $t \in[1, T]$,

$$
\begin{aligned}
\sum_{\nu=1}^{t} \mathbb{C}\left(\vec{x}^{p}(\nu), \vec{y}^{p}(\nu)\right) & \leq \sum_{\nu=1}^{t} \mathbb{C}\left(\vec{x}^{*}(\nu), \vec{y}^{*}(\nu)\right) \\
& +\sum_{\omega \in \Omega_{p, t}} C_{M G}^{\omega}\left(\vec{y}^{*}(\omega-1), \vec{y}^{(p)}(\omega-1)\right) .
\end{aligned}
$$

Proof sketch: $F H C^{(p)}$ solves (16) for locally-optimal routing and aggregation decisions in the time frame $[\omega, \omega+l]$. Total cost incurred by $F H C^{(p)}$ in $[\omega, \omega+l]$ is at most that of any strategy with the same initial aggregation decision $\vec{y}^{(p)}(\omega-1)$. Hence, the total cost incurred by $F H C^{(p)}$ in $[\omega, \omega+l]$ should be smaller than that of the following strategy: we first migrate the data from the data center specified by $\vec{y}^{(p)}(\omega-1)$ to that specified by $\vec{y}^{*}(\omega-1)$, and then operate data routing and aggregation in $[\omega, \omega+l]$ following the offline optimum solution in this time frame. We have

$$
\begin{aligned}
& \sum_{\nu=1}^{t} \mathbb{C}\left(\vec{x}^{p}(\nu), \vec{y}^{p}(\nu)\right) \\
&=\sum_{\omega \in \Omega_{p, t}} \sum_{\nu=\omega}^{\omega+l}\left\{C_{-M G}^{\nu}\left(\vec{x}^{(p)}(\nu), \vec{y}^{(p)}(\nu)\right)\right. \\
&\left.+C_{M G}^{\nu}\left(\vec{y}^{(p)}(\nu), \vec{y}^{(p)}(\nu-1)\right)\right\} \\
& \leq \sum_{\omega \in \Omega_{p, t}}\left\{\sum _ { \nu = \omega } ^ { \omega + l } \left\{C_{-M G}^{\nu}\left(\vec{x}^{*}(\nu), \vec{y}^{*}(\nu)\right)\right.\right. \\
&\left.\left.+C_{M G}^{\nu}\left(\vec{y}^{*}(\nu), \vec{y}^{*}(\nu-1)\right)\right\}+C_{M G}^{\omega}\left(\vec{y}^{*}(\omega-1), \vec{y}^{(p)}(\omega-1)\right)\right\} \\
&= \sum_{\nu=1}^{t} \mathbb{C}\left(\vec{x}^{*}(\nu), \vec{y}^{*}(\nu)\right)+\sum_{\omega \in \Omega_{p, t}} C_{M G}^{\omega}\left(\vec{y}^{*}(\omega-1), \vec{y}^{(p)}(\omega-1)\right) .
\end{aligned}
$$

Theorem 4. The RFHC algorithm is $\left(1+\frac{1}{l+1} \frac{\kappa}{\lambda}\right)$-competitive, where $\kappa=\sup _{t \in[1, T], \vec{y}^{1}(t), \vec{y}^{2}(t) \in \mathcal{Y}} \frac{C_{M G}^{t}\left(\vec{y}^{1}(t), \vec{y}^{2}(t)\right)}{\sum_{\nu=1}^{t-1}\left(\alpha_{\nu} \sum_{d \in \mathcal{D}} F_{d}(\nu)\right)}$ is the maximum migration cost per unit data, and $\lambda=$

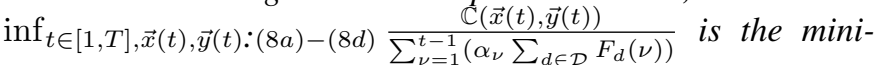
mum total cost per unit data per time slot.

Proof sketch: Let $C_{F H C^{(p)}}=\sum_{\nu=1}^{T} \mathbb{C}\left(\vec{x}^{p}(\nu), \vec{y}^{p}(\nu)\right)$ be the overall cost incurred by an $F H C^{(p)}$ algorithm, and $C_{O P T}=$ $\sum_{\nu=1}^{T} \mathbb{C}\left(\vec{x}^{*}(\nu), \vec{y}^{*}(\nu)\right)$ be the offline-optimal cost achieved by Alg. 1 . The expected cost of the RFHC algorithm is

$$
\begin{aligned}
& E(R F H C)=\frac{1}{l+1} \sum_{p=1}^{l+1} C_{F H C}(p) \\
\leq & \frac{1}{l+1} \sum_{p=1}^{l+1}\left\{C_{O P T}+\sum_{\omega \in \Omega_{p, t}} C_{M G}^{\omega}\left(\vec{y}^{*}(\omega-1), \vec{y}^{(p)}(\omega-1)\right)\right\} \\
= & C_{O P T}+\frac{1}{l+1} \sum_{p=1}^{l+1} \sum_{\omega \in \Omega_{p, t}} C_{M G}^{\omega}\left(\vec{y}^{*}(\omega-1), \vec{y}^{(p)}(\omega-1)\right) .
\end{aligned}
$$

The ratio of the above second term over $C_{O P T}$ is:

$$
\begin{aligned}
& \frac{\sum_{p=1}^{l+1} \sum_{\omega \in \Omega_{p, t}} C_{M G}^{\omega}\left(\vec{y}^{*}(\omega-1), \vec{y}^{(p)}(\omega-1)\right)}{C_{O P T}} \\
& =\frac{\sum_{\nu=1}^{T} C_{M G}^{\nu}\left(\vec{y}^{*}(\nu-1), \vec{y}^{(p)}(\nu-1)\right)}{\sum_{\nu=1}^{T} \mathbb{C}\left(\vec{x}^{*}(\nu), \vec{y}^{*}(\nu)\right)} \leq \frac{\kappa}{\lambda} .
\end{aligned}
$$


Therefore, we conclude that: $\frac{E(R F H C)}{C_{O P T}} \leq 1+\frac{1}{l+1} \frac{\kappa}{\lambda}$. $\square$ Theorem 4 reveals that the more future steps predicted (the larger $l$ is), the closer the RFHC algorithm can approach the offline optimal algorithm. Values of $\kappa$ and $\lambda$ are related to system input including prices and delays, and are less involved with the data generation patterns and the number of data centers. In our experiments, $\frac{\kappa}{\lambda} \approx 0.69$, and even with $l=1$, the competitive ratio is already as low as 1.34 . We also conducted experiments with imperfect predictions. Results show that the performance remains good when accuracy is above $80 \%$. When $l=0$, whether the RFHC algorithm or the lazy migration algorithm performs better depends on the system input. We will evaluate their performance through experiments in the next section.

Theorem 5. The RFHC algorithm has a worst-case computation complexity of

$$
\begin{cases}O\left(T \gamma D^{3} G^{3} K^{7} / \ln \left(D G K^{2}\right)\right) & \text { if } l=0 \\ O\left(T \gamma D^{3} G^{3} K^{8} / \ln \left(D G K^{2}\right)\right) & \text { if } l>0,\end{cases}
$$

Proof sketch:

Case 1: $l=0$ (no lookahead). In this case, at time slot $t$, the algorithm needs to go through the $K$ possible aggregation decisions of $\vec{y} \in \mathcal{Y}$ to derive the one-shot optimum. For each $\vec{y}$, the linear program in (10) is solved, with a computation complexity of $O\left(\gamma D^{3} G^{3} K^{6} / \ln \left(D G K^{2}\right)\right)$. Hence, the RFHC algorithm has a worst-case computation complexity of

$O\left(T \cdot \gamma D^{3} G^{3} K^{6} / \ln \left(D G K^{2}\right) \cdot K\right)=O\left(T \gamma D^{3} G^{3} K^{7} / \ln \left(D G K^{2}\right)\right)$.

Case 2: $l>0$. The algorithm computes the optimum of the data migration problem over the equal-size frames using the method introduced in Sec. IV. According to Theorem 1, the worst-case computation complexity of the RFHC algorithm in the first frame is $O\left((p-1) \gamma D^{3} G^{3} K^{8} / \ln \left(D G K^{2}\right)\right)$, and $O\left((l+1) \gamma D^{3} G^{3} K^{8} / \ln \left(D G K^{2}\right)\right)$ for each of the frames (of $l+1$ slots) afterwards. Hence, the RFHC algorithm has a worstcase computation complexity of

$$
\begin{aligned}
O & \left(\left\lfloor\frac{T-p}{l+1}\right\rfloor \cdot(l+1) \gamma D^{3} G^{3} K^{8} / \ln \left(D G K^{2}\right)\right) \\
& +O\left((p-1) \gamma D^{3} G^{3} K^{8} / \ln \left(D G K^{2}\right)\right) \\
= & O\left(T \gamma D^{3} G^{3} K^{8} / \ln \left(D G K^{2}\right)\right) .
\end{aligned}
$$

Theorem 5 shows the cumulative complexity from $t=1$ to $t=T$. Since RFHC is an online algorithm producing partial results in each time frame, we are more interested in the amortized per time frame complexity:

$$
\begin{cases}O\left(\gamma D^{3} G^{3} K^{7} / \ln \left(D G K^{2}\right)\right) & \text { if } l=0 \\ O\left((l+1) \gamma D^{3} G^{3} K^{8} / \ln \left(D G K^{2}\right)\right) & \text { if } l>0,\end{cases}
$$

\section{Performance Evaluation}

\section{A. Experiment Setup}

Astronomical data processing usually involves Terabytes, Petabytes, or even Exabytes of data per day. Square Kilometre Array (SKA) is an international project to build the world's largest telescope distributed in Australia, New Zealand and South Africa. It is expected to produce a few Exabytes of data per day [29]. Such astronomical data from geo-distributed telescopes is a natural target for our proposed algorithms.
We implement our offline and online algorithms on a cluster of 22 commodity computers (Intel(R) Pentium(R) D $3.0 \mathrm{GHz}$ with dual cores, $1 \mathrm{G}$ RAM). We emulate a cloud of 8 data centers in 8 regions (London, Bangalore, Hong Kong, Tokyo, San Francisco, Atlanta, Toronto and São Paulo), and a network of 8 observatories scattered in 8 time zones (Canary Islands, South Africa, Urumqi, Western Australia, New Zealand, Hawaii, Arizona, and Middle Chile), producing Gigabytes of astronomical data per night for upload into the cloud. There are 8 gateways collocated with the data centers and 6 user-side gateways in Paris, Beijing, Sydney, Los Angeles, New York and Rio de Janeiro, respectively. Each PC emulates one of user locations, user-side gateways and data centers. Networking instead of computation is our focus of emulation. Each PC can provide the ability to transfer a large amount of data via high-speed Ethernet. All 22 PCs are interconnected via a Dell PowerConnect 5548 Switch. An additional PC is employed to perform the central controller to control the routing schedule and the migration scheme within the cluster of 22 commodity computers. A typical cloud provider in practice usually has up to 8 data centers (e.g., Amazon EC2 consists of 8 data centers [30], Linode has 6 data centers [31] and DigitalOcean is operating 3 data centers [32].

Since detailed astronomical data are not publicly available, synthetic traces are generated to drive our experiments. We extract data generation patterns from a set of statistics on atmospheric visibility data collected at the Canarian observatories, from Feb. 2000 to Aug. 2003 [33]. Based on the strong correlation between the visibility and the quality of astronomical images [34], we produce astronomical data for each of the 8 observatories following the extracted data generation pattern, but augment the volume to the Gigabyte scale. Fig. 4 depicts the data patterns for two observatories, each producing data between 9:00 p.m. to 5:00 a.m. in its local time.

Each time slot is set to one hour in our experiments. Link delays are set based on Round Trip Time (RTT) among the observatories, gateways and data centers, according to geographic distance: $R T T(\mathrm{~ms})=0.02 \times \operatorname{Distance}(\mathrm{km})+5$ [35]. Bandwidths on the VPN connections are randomly drawn within $[68,100]$ Mbps. The unit charges of uploading data via the VPN connections, $f_{g i}$, are set within [0.10, 0.25] \$/GB. The unit cost for storing and processing one GB of data, $s_{k}$ and $v_{k}$ in data center $k$, are set within [0.01, 0.1] $\$ / \mathrm{h}$ and $[0.45,0.55] \$ / \mathrm{h}$ respectively, following typical charges in Amazon EC2 and S3 [30], [36] respectively, with small value variations. The rationale behind such setting of $v_{k}$ include: (i), TERAPIX [37], which is designed to handle large amounts of astronomical data, can process 320 GB data per night, using a cluster of 10 machines each configured with dual Athlon $\mathrm{XP} / \mathrm{MP} 2400+$ and 2 GB RAM; (ii), the measurement of EC2 instances [30]. From (i) and (ii) we estimate the unit cost for processing $1 \mathrm{~GB}$ data, and the algorithm will setup enough number of VMs to execute MapReduce according to the current data volume. The migration cost function, $\phi_{i k}(z)$ is a linear function on the amount of data moved, $z$, with a cost in the range of $[0.10,0.25] \$ / G B$. The default value of $L$ to convert delay to cost is 0.01 .

At time $t$, data produced in $[t-2, t]$ are used for processing, 


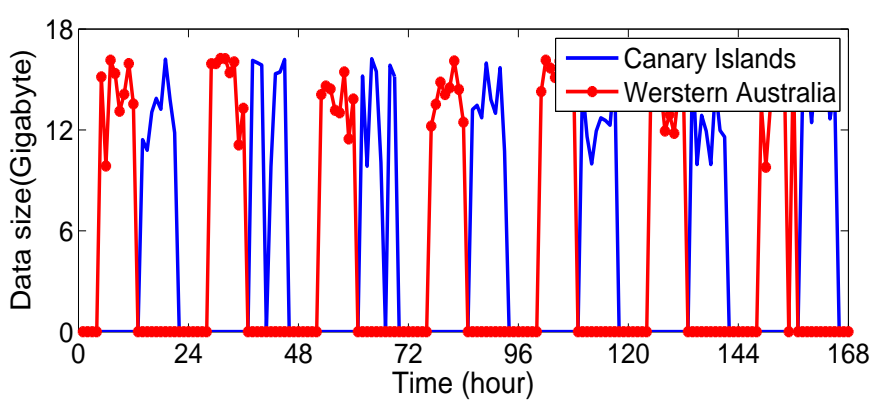

Fig. 4. Astronomical data generating pattern: 9:00 p.m. GMT+12 is the timeline starting point.

TABLE I

Constant PRices, $P=0.25, L=0.01$

\begin{tabular}{|l|l|l|l|l|l|}
\hline & Simple & OLM & RFHC(0) & RFHC(1) & Offline \\
\hline Overall cost (\$) & 20557 & 17264 & 18503 & 17237 & 16712 \\
\hline Ratio & 1.23 & 1.03 & 1.11 & 1.03 & 1 \\
\hline \multicolumn{5}{|c}{ TABLE II }
\end{tabular}

Constant Prices, $P=0.25, L=0.01$, ScAle UP THE UnIT COMPUTATION COST BY 10

\begin{tabular}{|l|l|l|l|l|l|}
\hline & Simple & OLM & RFHC(0) & RFHC(1) & Offline \\
\hline Overall cost (\$) & 57827 & 52742 & 53653 & 52904 & 52370 \\
\hline Ratio & 1.10 & 1.01 & 1.02 & 1.01 & 1 \\
\hline
\end{tabular}

i.e., $\alpha_{t}=1, \alpha_{t-1}=\alpha_{t-2}=P$, and $\alpha_{t-2}=\ldots=\alpha_{1}=0$, where $P$ is a parameter adjusted to investigate the impact of the volume of historical data involved.

We compare the costs incurred by the offline and online algorithms, as well as a simple algorithm that fixes the aggregation data center to the one in Hong Kong (relatively central to all observatories with moderate data center prices), and only computes the data uploading paths in each hour based on (10), similar to that in Choi and Gupta [16]. Default parameter values for OLM are $\beta_{1}=0.5, \beta_{2}=2$. Default lookahead window size for RFHC is $l=1$.

\section{B. Constant Prices}

We first evaluate the performance of the algorithms under the above default, fixed prices for data storage and processing in the data centers. In Tab. I, $R F H C(x)$ represents a RFHC algorithm with lookahead window $l=x$. As compared to the simple algorithm, all proposed algorithms achieve considerable cost reduction, and performance of the OLM algorithm and the RFHC algorithm with $l=1$ approach that of the offline optimum rather well. We also compare the performance between OLM and $\operatorname{RFHC}(0)$, which is in fact an algorithm to solve the one-shot optimization (11) in each time slot. Fig. 5 shows that $\mathrm{RFHC}(0)$ incurs higher costs, due to moving data back and forth, while OLM can prevent such costs.

We next scale the processing price by a factor of 10 and $\frac{1}{10}$, respectively, with other settings unchanged. Tab.II and Tab. III show that our online algorithms still outperform the simple algorithm, and demonstrate close-to-offline-optimum performance in both cases. This observation demonstrates the validity and effectiveness of OLM and RFHC.

\section{Dynamic VM pricing}

We next investigate dynamical VM prices (time-varying data processing costs) following the Spot Instance prices from

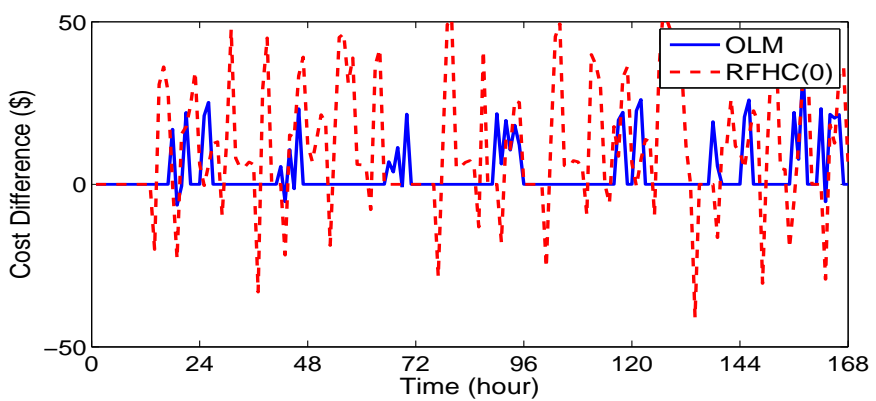

Fig. 5. Cost difference as compared to offline optimum: constant prices, $P=0.25, L=0.01$.

TABLE III

Constant PRices, $P=0.25, L=0.01$, SCALE DOWN THE Unit COMPUTATION COST BY 10

\begin{tabular}{|l|l|l|l|l|l|}
\hline & Simple & OLM & RFHC(0) & RFHC(1) & Offline \\
\hline Overall cost (\$) & 16380 & 13844 & 14824 & 13737 & 13138 \\
\hline Ratio & 1.25 & 1.05 & 1.13 & 1.03 & 1.05 \\
\hline
\end{tabular}

TABLE IV

PERFORMANCE COMPARISON AMONG THE ALGORITHMS: SPOT INSTANCE PRICING, $P=0.25, L=0.01$

\begin{tabular}{|l|l|l|l|l|l|}
\hline & Simple & OLM & RFHC(0) & RFHC(1) & Offline \\
\hline Overall cost (\$) & 24709 & 16870 & 16676 & 16327 & 15944 \\
\hline Ratio & 1.55 & 1.06 & 1.05 & 1.02 & 1 \\
\hline \multicolumn{7}{|c|}{ TABLE V }
\end{tabular}

PERFORMANCE OF RFHC ALGORITHMS WITH DIFFERENT LOOKAHEAD WINDOW SIZES: CONSTANT PRICES, $P=0.25, L=0.01$

\begin{tabular}{|l|l|l|l|l|l|}
\hline & RFHC(0) & RFHC(1) & RFHC(2) & RFHC(3) & Offline \\
\hline $\begin{array}{l}\text { Overall } \\
\text { cost (\$) }\end{array}$ & 18503 & 17237 & 17050 & 16828 & 16712 \\
\hline Ratio & 1.11 & 1.03 & 1.02 & 1.01 & 1 \\
\hline
\end{tabular}

TABLE VI

PERFORMANCE OF RFHC ALGORITHMS WITH DIFFERENT LOOKAHEAD WINDOW SIZES: SPOT INSTANCE PRICING, $P=0.25, L=0.01$

\begin{tabular}{|l|l|l|l|l|l|}
\hline & RFHC(0) & RFHC(1) & RFHC(2) & RFHC(3) & Offline \\
\hline $\begin{array}{l}\text { Overall } \\
\text { cost (\$) }\end{array}$ & 16676 & 16327 & 16105 & 16138 & 15944 \\
\hline Ratio & 1.05 & 1.02 & 1.01 & 1.01 & 1 \\
\hline
\end{tabular}

Amazon EC2 during Apr. 2012 to Jul. 2012. From Tab. IV and Fig. 6, we see that due to lack of future price information, the OLM algorithm performs slightly worse than the RFHC algorithm with lookahead window $l=1$.

\section{Different Sizes of Lookahead Window}

We evaluate the RFHC algorithm with different lookahead window sizes, both with constant and with dynamic prices. Tab. V and Tab. VI show that, with the increase of the lookahead window, the performance is approaching the offline optimum. Just a $1-2$ step 'peek' into the future drives the performance very close to the offline optimum.

\section{E. Different Routing Latency Ratios}

We further study different routing latency ratios in Eqn. 6. Intuitively, a larger weight of routing cost make the system evaluate the upload performance more seriously, while a smaller weight means monetary cost minimization has higher priority. We compare the average latency for different weights of routing cost, and find that the latency decreases when the weight increases (Tab. VII). Here we set moderate $L=0.01$ and lower weight $L=0.001$ respectively. Average latency is reduced with a large $L$. 


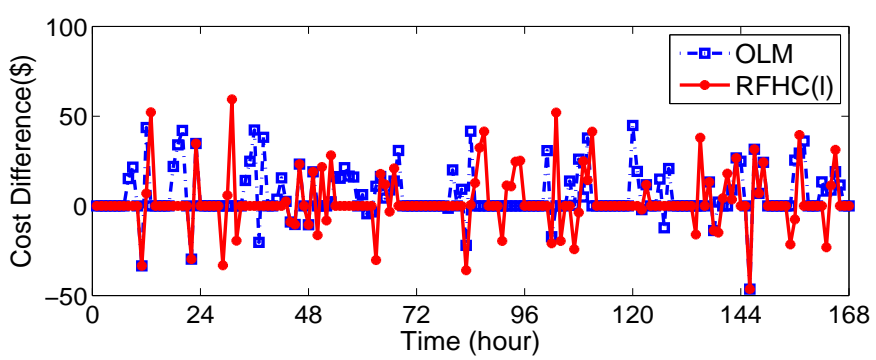

Fig. 6. Cost difference as compared to offline optimum over time: Spot Instance pricing, $P=0.25, L=0.01$.

TABLE VII

AVERAGE LATENCY OF EACH ALGORITHM: SPOT INSTANCE PRICING, $P=0.25$

\begin{tabular}{|c|l|l|l|l|l|l|}
\hline \multicolumn{2}{|c|}{ Algorithms } & Simple & OLM & RFHC(0) & RFHC(1) & Offline \\
\hline Average & $\begin{array}{l}L= \\
\text { latency }(m s)\end{array}$ & 224.2 & 165.3 & 177.0 & 169.2 & 166.5 \\
\cline { 2 - 7 } & $\begin{array}{l}L=01 \\
0.001\end{array}$ & 232.2 & 202.5 & 219.6 & 218.7 & 216.6 \\
\hline
\end{tabular}

\section{F. Involvement of Historical Data}

We next investigate the impact of weights of earlier data, under dynamic spot instance pricing. As compared to Tab. IV and Fig. 6, the results in Tab. VIII and Fig. 7 are derived under the same settings except a larger $P$, i.e., more historical data are involved in the computation and migration in each time slot. We observe that the performance of our algorithms slightly degrades. This can be explained as follows: when more historical data are involved, the migration cost is larger, and a wrong aggregation decision may incur higher increase in the cost. The outlier at $t=50$ in the curve of the OLM algorithm in Fig. 7 shows the cost of such a wrong decision.

TABLE VIII

PERFORMANCE COMPARISON AMONG THE ALGORITHMS: SPOT INSTANCE PRICING, $P=0.5, L=0.01$

\begin{tabular}{|l|l|l|l|l|l|}
\hline & Simple & OLM & RFHC(0) & RFHC(1) & Offline \\
\hline Overall cost (\$) & 27822 & 20884 & 19706 & 18889 & 17917 \\
\hline Ratio & 1.55 & 1.17 & 1.10 & 1.05 & 1 \\
\hline
\end{tabular}

\section{G. Impact of non-perfect prediction}

In Sec. V we assumed 100\% prediction accuracy. Next we investigate the impact of prediction errors by considering the following two configurations: (i), spot instance pricing, $P=$ $0.5, L=0.01$; (ii), spot instance pricing, $P=0.25, L=0.01$. We generate the predicted input with error through adjusting the accurate input from the trace by corresponding percentiles. Considering a wide range of prediction error rates $(40 \%, \ldots$, $10 \%, 0 \%$ ), we examine the performance of RFHC(1). As shown in Tab. IX, the RFHC algorithm performs better with lower prediction error rate. When the error rate is adequately low, the cost is very close to the one with $100 \%$ accurate prediction, sometimes even better, e.g., $10 \%$ in Tab. IX. That is possible because the aggregation decisions made by RFHC(1) with $100 \%$ accurate prediction are still myopic, maybe far from offline optimum. Small prediction error sometimes help the algorithm avoid shortsighted decisions. In addition, we observe that even with $40 \%$ prediction error rate, the performance is still better than $\operatorname{RFHC}(0)$.

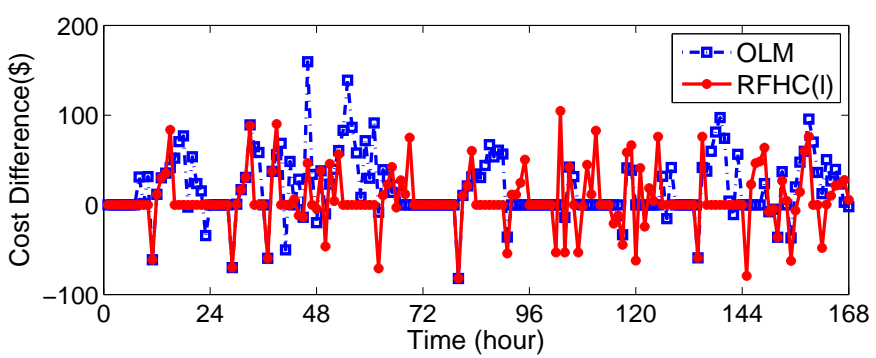

Fig. 7. Cost difference as compared to offline optimum over time: Spot Instance pricing, $P=0.5, L=0.01$.

TABLE IX

SPOT INSTANCE PRICING, $L=0.01, \operatorname{RFHC}(1)$

\begin{tabular}{|c|l|l|l|l|l|l|l|}
\hline \multicolumn{2}{|c|}{ Error rate } & $40 \%$ & $30 \%$ & $20 \%$ & $10 \%$ & $0 \%$ & RFHC(0) \\
\hline Overall & $\begin{array}{l}P= \\
0.5\end{array}$ & 19455 & 19423 & 19147 & 19111 & 18889 & 19706 \\
\cline { 2 - 8 } cost (\$) & $\begin{array}{l}P= \\
0.25\end{array}$ & 16516 & 16396 & 16328 & 16304 & 16327 & 16676 \\
\hline
\end{tabular}

\section{H. Execution time}

Algorithm running times are recorded as follows - OLM: $4.55 s$ per time slot; RFHC(0): $4.49 s$ per time slot; RFHC(1): $9.91 \mathrm{~s}$ per time frame. All algorithms finish within 10 seconds, which is small given the time slot length of 1 hour. RFHC(1) needs more time because the length of its time frame is 2 time slots. According to Eqn. 19, the running time of RFHC(1) is proportional to the length of time frame.The running time may be further reduced substantially through using powerful computing servers, optimized $\mathrm{C} / \mathrm{C}++$ implementation and more efficient linear program solver, e.g., glpk.

\section{CONCLUDING REMARKS}

This paper designs efficient algorithms for timely, costminimizing migration of geo-dispersed big data to the cloud, for processing using a MapReduce-like framework. A polynomial-time optimal offline algorithm is proposed based on dynamic programming. Two online algorithms are designed to practically guild data migration in an online fashion: the OLM algorithm achieves a worst-case competitive ratio as low as 2.55 under typical real-world settings; the RFHC algorithm provides a decreasing competitive ratio with an increasing size of the lookahead window.

\section{REFERENCES}

[1] Rackspace, http://www.rackspace.com/

[2] M. Armbrust, A. Fox, R. Grifth, A. D. Joseph, R. Katz, A. Konwinski, G. Lee, D. P. A. Rabkin, I. Stoica, and M. Zaharia, "Above the Clouds: A Berkeley View of Cloud Computing," EECS, University of California, Berkeley, Tech. Rep., 2009.

[3] S. Pandey, L. Wu, S. Guru, and R. Buyya, "A Particle Swarm Optimization (PSO)-based Heuristic for Scheduling Workflow Applications in Cloud Computing Environment," in Proc. of IEEE AINA, 2010.

[4] Human Genome Project, http://www.ornl.gov/hgmis/home.shtml.

[5] Hadoop - Facebook, http://www.facebook.com/note.php?note_id=1612 1578919.

[6] Hadoop at Twitter, http://www.slideshare.net/kevinweil/hadoop-attwitter-hadoop-summit-201.

[7] AWS Import/Export, http://aws.amazon.com/importexport/.

[8] E. E. Schadt, M. D. Linderman, J. Sorenson, L. Lee, and G. P. Nolan, "Computational Solutions to Large-scale Data Management and Analysis," Nat Rev Genet, vol. 11, no. 9, pp. 647-657, 092010. 
[9] Moving an Elephant: Large Scale Hadoop Data Migration at Facebook, http://www.facebook.com/notes/paul-yang/moving-an-elephant-largescale-hadoop-data-migration-at-facebook/10150246275318920.

[10] R. J. Brunner, S. G. Djorgovski, T. A. Prince, and A. S. Szalay, "Handbook of Massive Data Sets," J. Abello, P. M. Pardalos, and M. G. C. Resende, Eds. Norwell, MA, USA: Kluwer Academic Publishers, 2002, ch. Massive Datasets in Astronomy, pp. 931-979.

[11] M. Cardosa, C. Wang, A. Nangia, A. Chandra, and J. Weissman, "Exploring MapReduce Efficiency with Highly-Ditributed Data," in Proc. of ACM MapReduce, 2011.

[12] Amazon Elastic MapReduce, http://aws.amazon.com/elasticmapreduce/.

[13] M. Hajjat, X. Sun, Y. E. Sung, D. Maltz, and S. Rao, "Cloudward Bound: Planning for Beneficial Migration of Enterprise Applications to the Cloud," in Proc. of ACM SIGCOMM, August 2010.

[14] X. Cheng and J. Liu, "Load-Balanced Migration of Social Media to Content Clouds," in Proc. of ACM NOSSDAV, June 2011.

[15] Y. Wu, C. Wu, B. Li, L. Zhang, Z. Li, and F. Lau, "Scaling Social Media Applications into Geo-Distributed Clouds," in Proc. of IEEE INFOCOM, Mar. 2012.

[16] B. Cho and I. Gupta, "New Algorithms for Planning Bulk Transfer via Internet and Shipping Networks," in Proc. of IEEE ICDCS, 2010.

[17] B. Cho and I. Gupta, "Budget-Constrained Bulk Data Transfer via Internet and Shipping Networks," in Proc. of ACM ICAC, 2011.

[18] M. Lin, A. Wierman, L. L. Andrew, and E. Thereska, "Dynamic Right-sizing for Power-proportional Data Centers," in Proc. of IEEE INFOCOM, April 2011.

[19] V. Mathew, R. Sitaraman, and P. Shenoy, "Energy-aware Load Balancing in Content Delivery Networks," in Proc. of IEEE INFOCOM, Mar. 2012.

[20] T. Lu and M. Chen, "Simple and Effective Dynamic Provisioning for Power-Proportional Data Centers," in Proc. of IEEE CISS, Mar. 2012.

[21] M. Lin, Z. Liu, A. Wierman, and L. Andrew, "Online Algorithms for Geographical Load Balancing," in Proc. of IEEE IGCC, 2012.

[22] AWS Direct Connect, http://aws.amazon.com/directconnect/.

[23] Windows Azure Virtual Network, http://www.windowsazure.com/enus/home/features/networking/.

[24] GoGrid, http://www.gogrid.com/.

[25] D. Logothetis, C. Olston, B. Reed, K. C. Webb, and K. Yocum, "Stateful Bulk Processing for Incremental Analytics," in Proc. of ACM SoCC, 2010.

[26] A. Borodin and R. El-Yaniv, Online Computation and Competitive Analysis. Cambridge University Press, 1998, vol. 2.

[27] K. M. Anstreicher, "Linear Programming in $O([n 3 / \ln n] L)$ Operations," SIAM Journal on Optimization, vol. 9, no. 4, pp. 803-812, 1999.

[28] G. E. P. Box, G. M. Jenkins, and G. C. Reinsel, Time Series Analysis: Forecasting and Control, 4th ed. Wiley, 2008.

[29] Square Kilometre Array, http://www.skatelescope.org/.

[30] Amazon Elastic Compute Cloud, http://aws.amazon.com/ec2/.

[31] Linode, https://www.linode.com/speedtest/.

[32] DigitalOcean, https://www.digitalocean.com/.

[33] Sky Quality Group, The Instituto de Astrofisica de Canarias (IAC), http://www.iac.es/proyecto/site-testing/index.php.

[34] H. V. Ramió, C. M. noz Tunón, A. M. Varela, and J. J. Fuensalida, "Complementary note: DIMM (ELT-DS) calibration," The Instituto de Astrofísica de Canarias (IAC), Tech. Rep., 2008.

[35] A. Qureshi, "Power-Demand Routing in Massive Geo-Distributed Systems," PhD Thesis submitted to MIT, 2010.

[36] Amazon Simple Storage Service, http://aws.amazon.com/s3/.

[37] TERAPIX, http://terapix.iap.fr/.

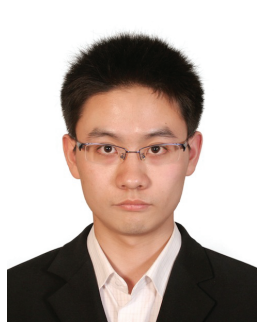

Linquan Zhang received his B.E. degree in 2010 from the Department of Computer Science and Technology, Tsinghua University, China, and his M.Phil. degree in 2012 from the Department of Computer Science, the University of Hong Kong. Since September, 2012, he has been with the Department of Computer Science, at the University of Calgary, Calgary, Canada, where he is currently a $\mathrm{Ph} . \mathrm{D}$. student. His research interests are mainly in cloud computing, network optimization and game theory.

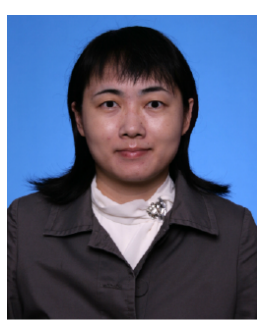

Chuan Wu received her B.E. and M.E. degrees in 2000 and 2002 from Department of Computer Science and Technology, Tsinghua University, China, and her Ph.D. degree in 2008 from the Department of Electrical and Computer Engineering, University of Toronto, Canada. She is currently an assistant professor in the Department of Computer Science, the University of Hong Kong, China. Her research interests include cloud computing, peer-to-peer networks and online/mobile social network. She is a member of IEEE and ACM.

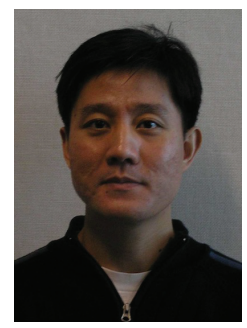

Zongpeng Li received his B.E. degree in Computer Science and Technology from Tsinghua University in 1999, his M.S. degree in Computer Science from University of Toronto in 2001, and his Ph.D. degree in Electrical and Computer Engineering from University of Toronto in 2005. Since August 2005, he has been with the Department of Computer Science in the University of Calgary. In 2011-2012, Zongpeng was a visitor at the Institute of Network Coding, CUHK. His research interests are in computer networks and network coding.

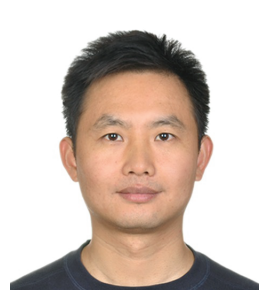

Chuanxiong Guo is a Principal Development Lead in the Windows Azure Group of Microsoft. Before that, he was a Senior Researcher in the Wireless and Networking Group of Microsoft Research Asia (MSRA). He received his Ph.D. degree from the Institute of Communications Engineering in Nanjing China. His research interests include networked systems design and analysis, network security, data centric networking, networking support for operating systems. He is currently working on data center networking (DCN) and Cloud Computing.

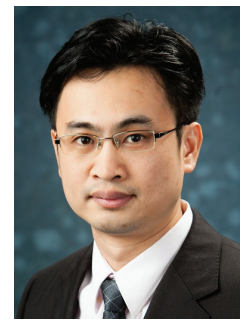

Minghua Chen received his B.Eng. and M.S. degrees in Electronic Engineering from Tsinghua University in 1999 and 2001, respectively, and his Ph.D. degree in Electrical Engineering and Computer Sciences from UC Berkeley in 2006. He spent one year visiting Microsoft Research Redmond as a Postdoc Researcher. He joined the Dept. of Information Engineering, the Chinese University of Hong Kong in 2007, where he is currently an Associate Professor. $\mathrm{He}$ is also an Adjunct Associate Professor in Peking University Shenzhen Graduate School in 2011-2014. He received the Eli Jury Doctoral Thesis award from UC Berkeley in 2007, the ICME Best Paper Award in 2009, the IEEE Trans. on Multimedia Best Paper Award in 2009, and the ACM Multimedia Best Paper Award in 2012. His current research interests include smart (micro) grids, energy-efficient data centers, distributed and stochastic network optimization and control, multimedia networking, p2p networking, wireless networking, network coding, and secure network communications.

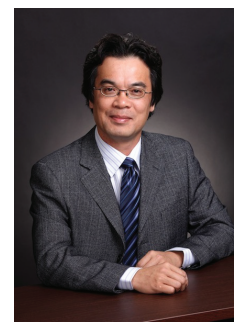

Francis C.M. Lau (SM) received a $\mathrm{PhD}$ in computer science from the University of Waterloo in 1986. He has been a faculty member of the Department of Computer Science, The University of Hong Kong since 1987, where he served as the department chair from 2000 to 2005 . He was a honorary chair professor in the Institute of Theoretical Computer Science of Tsinghua University from 2007 to 2010. His research interests include computer systems and networking, algorithms, HCI, and application of IT to arts. 